\title{
Droplet size measurement of liquid atomization by the immersion liquid method (droplet coalescence and solution into the immersion liquid)
}

\author{
T. Fujimatsu, M. Kito \& K. Kondo \\ Department of Mechanical Engineering, \\ Suzuka National College of Technology, Japan
}

\begin{abstract}
The influence of measurement methods with some measuring conditions on droplet coalescence and solution were experimentally investigated to measure the droplet size more accurately by the immersion liquid method. In the case of the sedimentation method, the experimental result shows that captured droplets coalesce and dissolve even in the immersion liquid as well as on its surface. It was also found that the solution of captured droplets into the immersion liquid considerably decreases by interrupting its surface from the air. Moreover, the influence of the immersion liquid viscosity on the droplet coalescence can be neglected within the very short shutter opening period (TS $\leq 103 \mathrm{~ms})$ irrespective of the sedimentation method or the hanging one. So, it is desirable to measure the droplet size as soon as possible after the droplets were completely immersed into the low viscosity immersion liquid, to prevent the effects of the droplet coalescence and solution into the immersion liquid.

Keywords: immersion liquid method, sedimentation method, hanging method, measurement technique, droplet coalescence, droplet solution, Sauter mean diameter.
\end{abstract}

\section{Introduction}

Immersion liquid method, impression method, direct photography method and solidifying method have been reported as the droplet size measurement $[1,2]$. Droplet size measurements using laser beam are also reported in these years [3]. 
Although the non-contact optical method provides much information on droplet size measurement in a short time, it requires occasional confirmations and collections to ascertain the measurement accuracy. The immersion liquid method is the classic calibration for the optical method [4].

In the immersion liquid method, liquid droplets are captured onto an immersion liquid surface within a glass plate or a shallow vessel, and a microscope is used to obtain magnified photographs of the droplets in the immersion liquid for droplet size measurement. This method is often used for the case where fuel or the like is sprayed out into an open space, since the method is cost-efficient and easy to use, and is one of the most fundamental, mechanical techniques to measure droplet sizes and distributions. For instance, the method is used to measure the sizes of droplets generated by swirl atomizers or rotating jets, ultrasonic vibrations, imitated disturbance waves, dispersed droplets in gasliquid two phase flow [5]. Hiroyasu [6] and Kurabayashi [7], however, have raise arguments with measurement accuracy caused by droplet coalescence, evaporation and disintegration on the immersion liquid surface. Therefore it has been studied that the conditions of the immersion liquid, the effects of droplet coalescence, evaporation and disintegration on its surface and a fine droplet escape during inserting a collector.

The authors have measured the size of water droplets generated from twofluid nozzle and ultrasonic humidifier by the immersion method using siliconeoil as the immersion liquid, and investigated the measurement accuracy, universality and repeatability. However, the effects of droplet coalescence and solution into the immersion liquid on the measurement accuracy are still unknown.

The authors present the effects of droplet coalescence and solution into silicone-oil on the accuracy of the droplet size measurement focusing on measuring the size of water droplets generated by ultrasonic vibration using the immersion liquid method. The effects of droplet solution into silicone-oil are investigated to establish a measurement method which is little influenced by the droplet solution for different immersion liquid types. Using the suggested method, the experiment was conducted to demonstrate the effects of droplet coalescence on the measurement accuracy varying the shutter opening period and silicone-oil viscosity.

\section{Experimental apparatus and procedure}

Figure 1 shows a schematic diagram of the experimental apparatus. Spray droplets generated by an ultrasonic humidifier (National, FE-05KYC) were jetted into still air through a $4 \mathrm{~mm}$ diameter nozzle. Downward liquid droplets generated from the nozzle are then captured by the sampling collector which consists of $39 \mathrm{~mm}$ outer diameter. An aperture with a diameter of $4 \mathrm{~mm}$ was drilled on an outer casing of cylindrical droplet sampling collector which is mounted a shutter and a sampling rod inside. When the shutter moves as a stopper is removed and the aperture on the shutter matches with the outer casing aperture, spray droplets are captured in a sampling tank $(4 \mathrm{~mm} \times 8 \mathrm{~mm}$ area and 


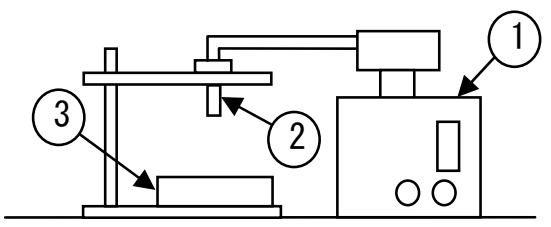

(a) Sampling system

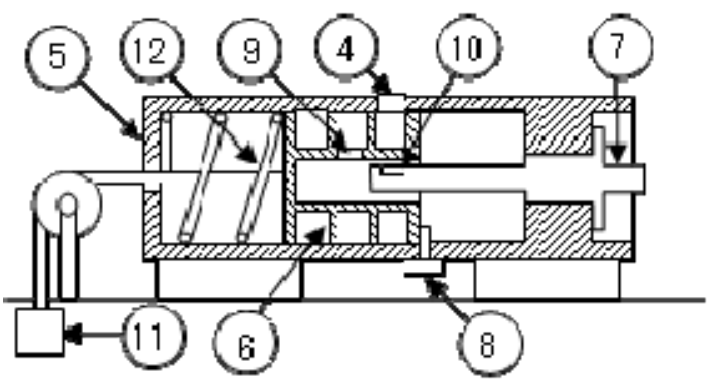

(b) Details of droplet sampling collector

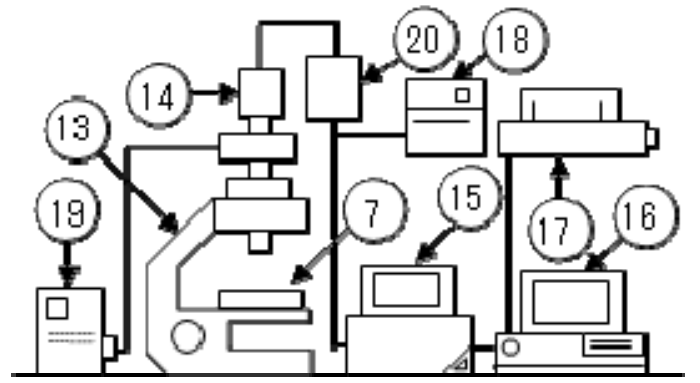

(c) Droplet size measurement system

$\begin{array}{ll}\text { 1: Ultrasonic humidifier } & \text { 11: Weight } \\ \text { 2: Nozzle } & \text { 12: Spring } \\ \text { 3: Droplet sampling collector } & \text { 13: Microscope } \\ \text { 4: Outer casing aperture } & \text { 14: CCD camera } \\ \text { 5: Outer casing } & \text { 15: Flexible image processor } \\ \text { 6: Shutter } & \text { 16: Desktop computer } \\ \text { 7: Sampling rod } & \text { 17: Printer } \\ \text { 8: Stopper } & \text { 18: Graphic printer } \\ \text { 9: Shutter aperture } & \text { 19: Light source } \\ \text { 10: Sampling tank } & \text { 20: Video control unit }\end{array}$

Figure 1: Schematic diagram of experimental apparatus.

$1.5 \mathrm{~mm}$ depth) coated with silicone-oil. The shutter opening period Ts is adjusted by a weight and a spring to demonstrate the effects of droplet coalescence and changed from 8 to $453 \mathrm{~ms}$. The kinematic viscosity of the immersion liquid varied from 10 to $5 \times 10^{4} \mathrm{~mm}^{2} / \mathrm{s}$. The distance between the nozzle edge of the ultrasonic humidifier and the silicone-oil surface was set to be $80 \mathrm{~mm}$. The nozzle was adjusted to coincide with the central axis of the hole in the 
collector. The droplet velocity at the collector was measured to be $u_{m}=9.0 \mathrm{~m} / \mathrm{s}$ by a high-speed video camera (nac, HSV-400).

The $410 \times 410 \mu \mathrm{m}$ images of the spray droplets captured on the silicone-oil surface were recorded, through the microscope and CCD camera mounted on the central axis of the nozzle, into the image processing system (ADS, PIP-4000) and used to calculate the mean diameter of the spray droplets: the Sauter mean diameter which is defined by

$$
D_{32}=\frac{\sum N_{i} D_{i}^{3}}{\sum N_{i} D_{i}^{2}}
$$

where $D_{i}$ and $N_{i}$ are the reference diameter and the number of captured droplets, respectively.

The uncertainty in calculating the mean value from the population occurs apart from the uncertainty in the measurement method. Nukiyama and Tanazawa [8] estimated that the uncertainty was within about $8 \%$ when the sample number was about 100 for uniform droplets and 500-1000 for non-uniform droplets. Therefore, over 2000 droplets were captured.

\section{Experimental results and discussion}

\subsection{Effects of droplet solution on the accurate measurements of droplet size}

\subsubsection{Comparison between sedimentation method and hanging method}

In this section, the hanging method in which droplets are on the immersion liquid (this is known as a general method) and the sedimentation method in which droplets are in the immersion liquid are compared.

Figure 2(a) shows the variations in the droplet number density $N_{i} / N_{0}$ (the ratio of the number of droplets $N_{i}$ in a certain time to the number of droplets $N_{0}$ when the first image was captured at $T_{E C}=0 \mathrm{~s}$ ) with the time elapsed $T_{E C}$ in the hanging method. The droplet number density $N_{i} / N_{0}$ reduced to less than a half at $T_{E C}=30 \mathrm{~s}$ and zero at $T_{E C} \geq 120 \mathrm{~s}$ for each silicone-oil viscosity $v_{T}$ and shutter opening period $T s$ due to droplet evaporation. The variations in $N_{i} / N_{0}$ with $T_{E I}$ in the sedimentation method are plotted in figure 2 (b). The time when droplets are completely immersed to the bottom of the immersion liquid is defined as $T_{E I}=0 \mathrm{~s}$.

The $N_{i} / N_{0}$ decreases in both methods, however it decreases more slowly than that in the hanging method. In addition, $N_{i} / N_{0}$ becomes zero at $T_{E I}=180 \mathrm{~s}$ for each condition since all captured droplets slowly dissolve.

Figure 3 presents the Sauter mean diameter $D_{32}$ with the time elapsed corresponding to the results in figure 2. The Sauter mean diameter $D_{32}$ decreases with increasing $T_{E C}$ as shown in figure 3(a). In the case of the large $T S$ ( $T_{S} \geq 123 \mathrm{~ms}$ ), the captured droplets are slowly evaporated since the large amount of droplets is captured on the silicone-oil at $T_{E C}=0 \mathrm{~s}$ resulting in droplet coalescence and large $D_{32}$. 


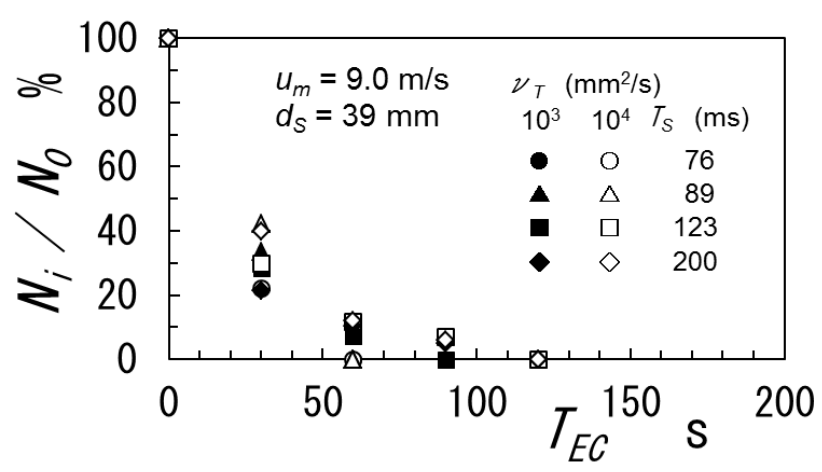

(a) Hanging method

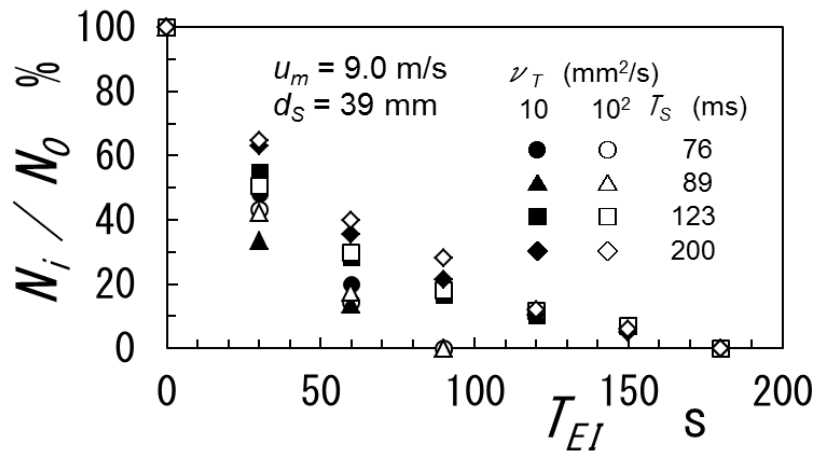

(b) Sedimentation method

Figure 2: Influence of measurement methods on droplet number density $\mathrm{Ni} / \mathrm{N}_{0}$ with time elapsed.

In the case of the sedimentation method, $D_{32}$ decreases as increasing $T_{E I}$ in a similar way of $N_{i} / N_{0}$ as shown in figure 3(b). However, more time elapsed is required to reach $D_{32}=0 \mu \mathrm{m}$ although the $D_{32}$ is almost same at $T_{E I}=0 \mathrm{~s}$.

The sedimentation method can suppress a reduction in droplet number and size. Still, droplet solution into the silicone-oil should be investigated varying the immersion liquids and measurement procedures.

\subsubsection{Examination of immersion liquids and measurement procedures}

The effects of droplet solution using silicone-oil saturated with water (hereinafter referred to as Type $S_{S}$ ) and unsaturated (hereinafter referred to as Type $S_{B}$ ) on the measurement accuracy were investigated in this section, since the captured droplets might dissolve into the silicone-oil.

Figure 4 shows the variations in $N_{i} / N_{0}$ and $D_{32}$ for different immersion liquids with the time elapsed $T_{E I}$ starting from when droplets were completely immersed to the bottom of the immersion liquid. Both $N_{i} / N_{0}$ and $D_{32}$ decrease over time, which is irrespective of the difference of immersion liquids. In the case of Type $\mathrm{S}_{\mathrm{B}}$, the captured droplets completely dissolve at $T_{E I}=90 \mathrm{~s}$, and $N_{i} / N_{0}$ and $D_{32}$ become zero. In the case of Type $\mathrm{S}_{\mathrm{S}}$, the captured droplets exist up to $T_{E I}=150 \mathrm{~s}$ 


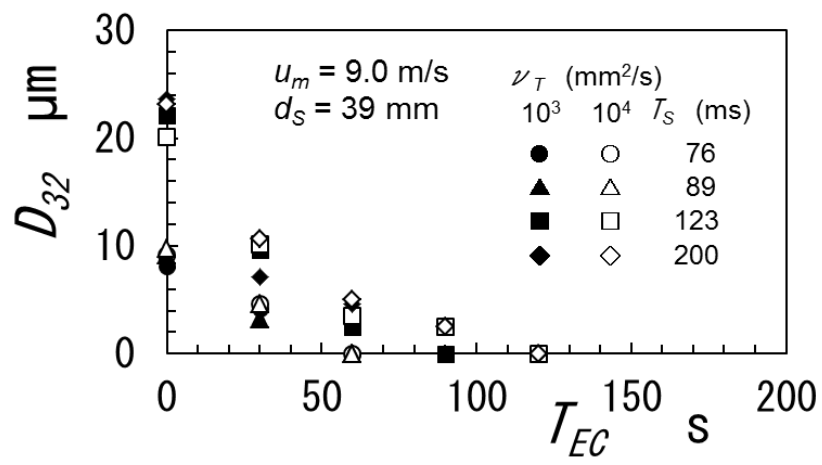

(a) Hanging method

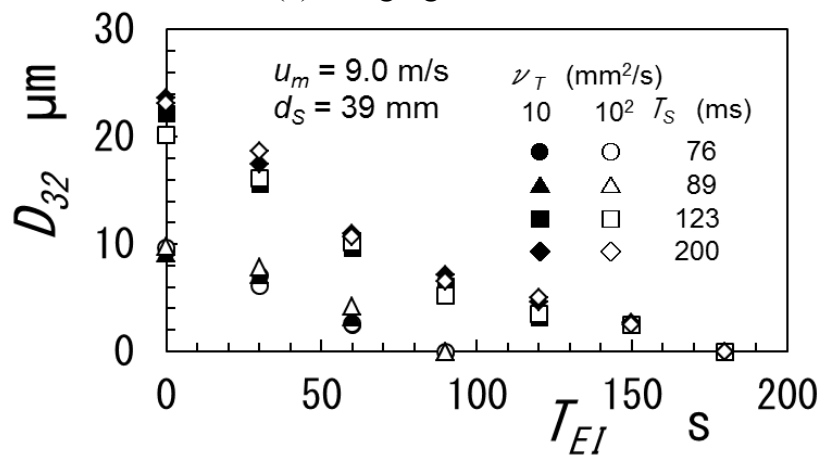

(b) Sedimentation method

Figure 3: Influence of measurement methods on Sauter mean diameter $D_{32}$ with time elapsed.

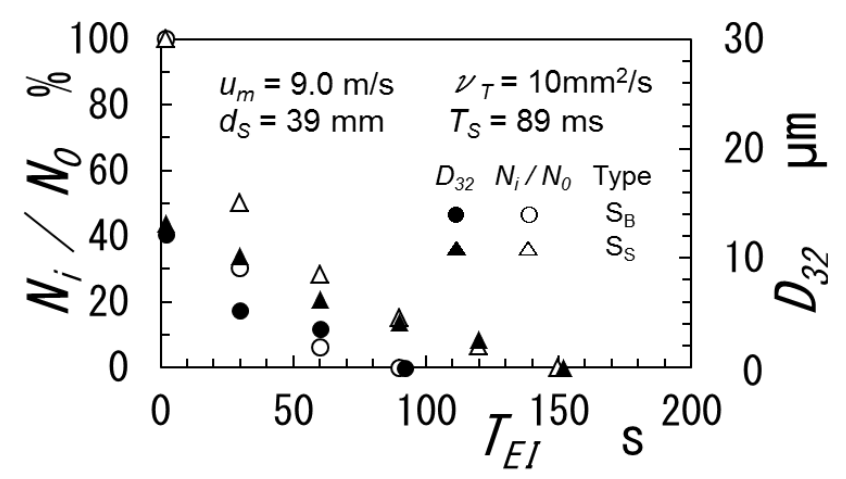

Figure 4: Influence of immersion liquids on $\mathrm{Ni} / \mathrm{N}_{0}$ and $\mathrm{D}_{32}$ with time elapsed $T_{E I}$ after complete immersion. 
in the immersion liquid. This is attributed to that the droplets exist longer in the oil because the Type $S_{S}$ is saturated with water and few droplets could dissolve into the oil. Therefore in the next experiment, the Type $\mathrm{S}_{\mathrm{S}}$ was used as the immersion liquid. The effects of droplet solution were investigated to establish the measurement procedure. The measurement procedures: Case A-Case D were conducted as shown in figure 5. The Case A is the traditional procedure in the immersion method. The Case B prevents the temperature of the silicone-oil from rising by tuning off the light except the time when images are captured. The Case $\mathrm{C}$ is basically the same as the Case A, except applying the water saturated oil on the collector after droplets were captured in order to immerse the droplets into the oil as soon as possible. The Case D is that a cover glass is put on the oil to avoid contact with ambient air after droplets were captured.

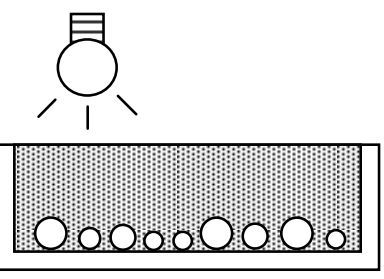

(a) Case A

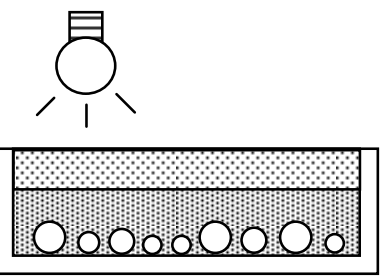

(c) Case C

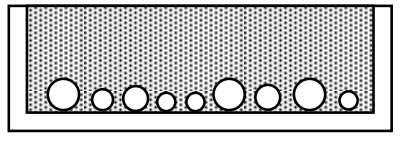

(b) Case B

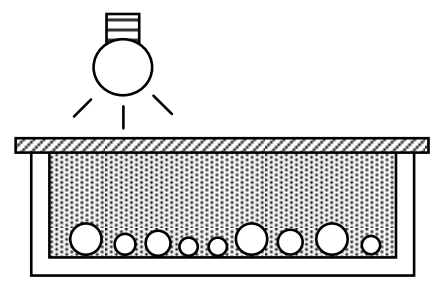

(d) Case D

Figure 5: Measurement techniques.

Figure 6 depicts the variations in $N_{i} / N_{0}$ for different measurement procedures. The time elapsed $T_{E I}$ is starting from the time when droplets were completely immersed to the bottom of the immersion liquid. In the Case A and Case B, $N_{i} / N_{0}$ decreases rapidly with time and becomes zero at $T_{E I}=150 \mathrm{~s}$. In Case C, $N_{i}$ $/ N_{0}$ gradually decreases, so that droplets in silicone-oil exist up to $T_{E I}=3000 \mathrm{~s}$. In the Case D, $N_{i} / N_{0}$ remains about $90 \%$ even after one hour, which indicates that droplet solution is suppressed significantly.

Figure 7 shows the Sauter mean diameter $D_{32}$ in the same manner as figure 6 . The Sauter mean diameter $D_{32}$ decreases rapidly as increasing $T_{E I}$ in the Case A and Case B. In the Case $\mathrm{C}$, after $D_{32}$ decreases slowly at $T_{E I} \leq 1800 \mathrm{~ms}$, the decreasing rate becomes large over time. In the Case $\mathrm{D}, D_{32}$ is little changed in one hour. 


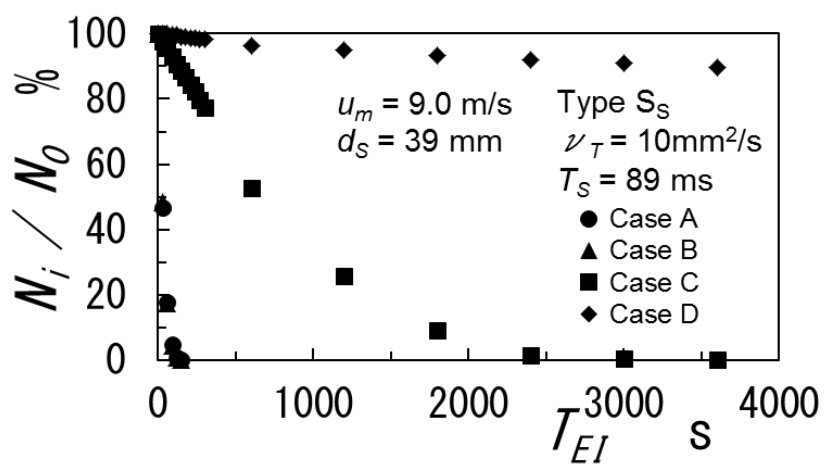

Figure 6: Influence of measurement techniques on $\mathrm{Ni} / \mathrm{N}_{0}$ with $T_{E I}$.

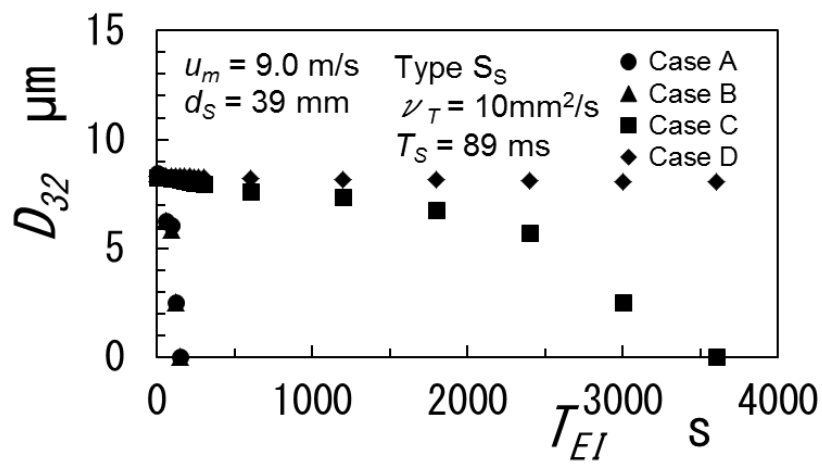

Figure 7: Influence of measurement techniques on $D_{32}$ with $T_{E I}$.

\subsection{Effects of droplet coalescence on the droplet-size measurement accuracy}

In this section, using the sedimentation method (Type Ss-Case D) with the best performance as mentioned in the section 3.1 , the effects of droplet coalescence in the silicone-oil were investigated varying the shutter opening period $T s$ and the silicone-oil viscosity $v_{T}$. A comparison between the traditional method (the hanging method, Type $\mathrm{S}_{\mathrm{B}}$-Case $\mathrm{A}$ ) and the presented method (the sedimentation method, Type $\mathrm{S}_{\mathrm{S}}-$ Case $\mathrm{D}$ ) is also shown.

\subsubsection{Effects of the shutter opening period on the Sauter mean diameter}

Figure 8 demonstrates the effects of the shutter opening period on the Sauter mean diameter comparing between the sedimentation method (Type $\mathrm{S}_{\mathrm{S}^{-}}$ Case D) and the hanging method (Type $\mathrm{S}_{\mathrm{B}}$-Case $\mathrm{A}$ ). In the case of the sedimentation method, while $D_{32}$ is approximately constant independently of the silicone-oil viscosity $v_{T}$ at $T s \leq 103 \mathrm{~ms}, D_{32}$ increases with $T s$ at $T s>103 \mathrm{~ms}$. This is attributed to droplet coalescence in the oil because the droplet number increases with $T s$. In the case of the hanging method, $D_{32}$ starts to increase at $T s$ 
$=100 \mathrm{~ms}$ and the increase rate of $D_{32}$ with $T s$ at $T s>100 \mathrm{~ms}$ is smaller than that of the sedimentation method. Although the coalescence of liquid droplet decreases as the shutter opening period $T s$ decreases, in this method, one must capture as many liquid droplets as possible to maintain measurement accuracy. It is therefore important to grasp the most suitable value of local droplet number per unit area $\delta_{L}$ (the number of captured droplets per unit area which is calculated from the all sampling images).

Figure 9 shows the effects of the local droplet number per unit area $\delta_{L}$ on the Sauter mean diameter $D_{32}$. In the sedimentation method, the value of $D_{32}$ is almost constant up to $\delta_{L} \cong 2000$, while the value of $D_{32}$ increases rapidly and $\delta_{L}$ decreases due to droplet coalescence at $\delta_{L}>2000$. The droplet coalescence in the silicone-oil can be diminished when the shutter opening period $T s$ is shorter than the time it takes for $\delta_{L}$ to become its maximum value. In the hanging method, a maximum of $\delta_{L}$ is about 3500 indicating that more droplets are captured than that in the sedimentation method. This suggests that droplet coalescence in the sedimentation method occurs more than that in the hanging method, which can be inferred from the $D_{32}$ value in figure 8 .

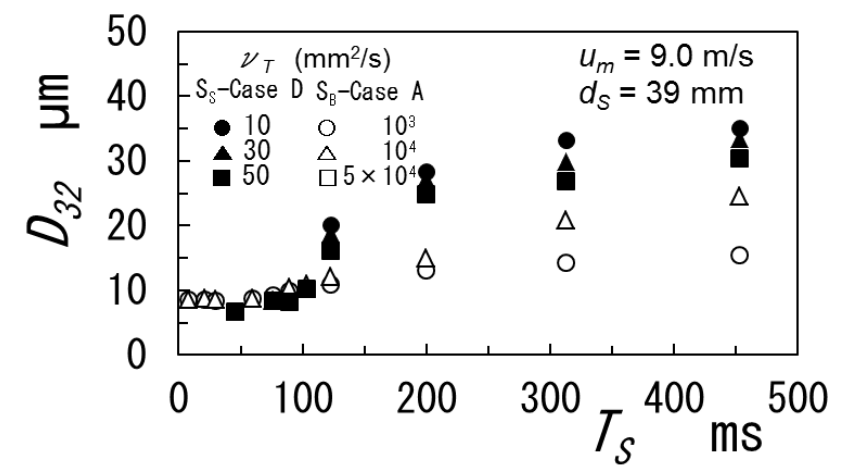

Figure 8: $\quad$ Influence of shutter opening period $T_{S}$ on $D_{32}$.

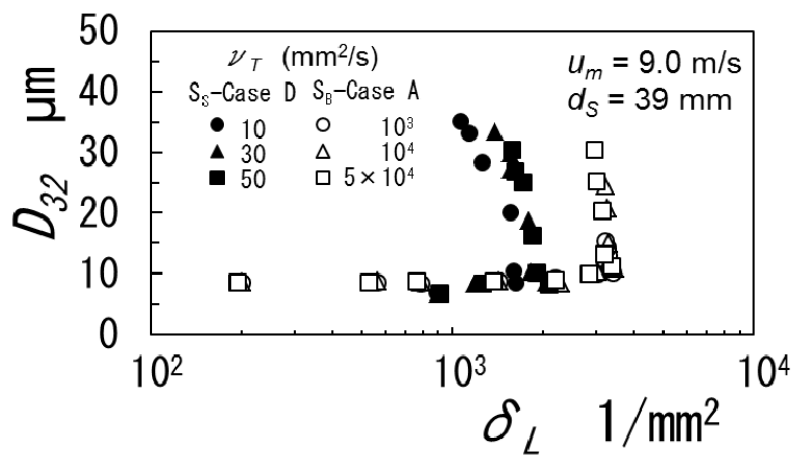

Figure 9: $\quad$ Change in $D_{32}$ with local droplet number per unit area $\delta_{L}$. 
Figure 10 shows the effects of the droplet area fraction $A_{F}$ (The area ratio of the droplets occupied to the measurement area) on the Sauter mean diameter $D_{32}$. In the case of the sedimentation method, while $D_{32}$ is almost constant at $A_{F}<5 \%$, $D_{32}$ increases with $A_{F}$ at $A_{F}>5 \%$, which is supported by Tate's statement [12] that the limitation of droplet rate is 0.05 regarding droplet coalescence. In the case of the hanging method, $D_{32}$ is almost constant at $A_{F}<10 \%$, which implies the hanging method can collect more droplets than the sedimentation method. The difference in the number of droplets for the different methods might be caused by the deformations and fluctuations on the silicone-oil surface due to the droplet impingement. Then, droplets coalesce, since the droplets float and move easily during sedimentation.

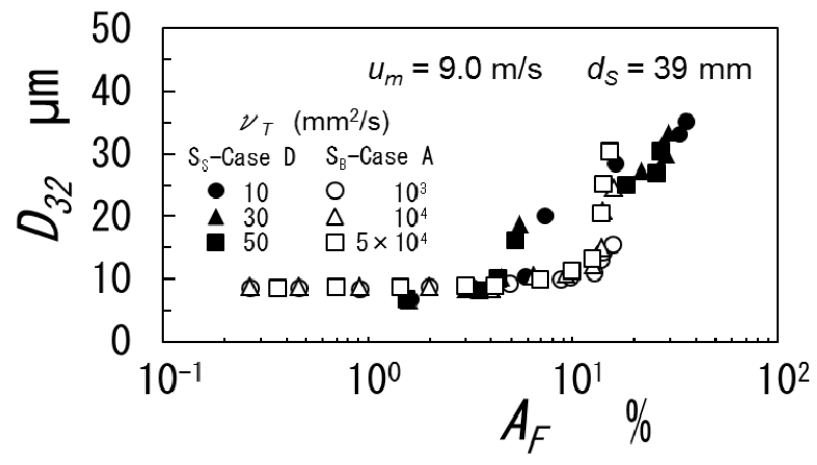

Figure 10: Change in $D_{32}$ with droplet area fraction $A_{F}$.

\subsubsection{Effects of silicone-oil viscosity on Sauter diameter}

Figure 11 shows the effects of the silicone-oil viscosity $v_{T}$ on the Sauter mean diameter $D_{32}$ measured with the hanging method $\left(10^{3} \mathrm{~mm}^{2} / \mathrm{s} \leq v_{T} \leq 5 \times 10^{4}\right.$ $\left.\mathrm{mm}^{2} / \mathrm{s}\right)$ together with the results using the sedimentation method $\left(1 \mathrm{~mm}^{2} / \mathrm{s}\right.$ $\leq v_{T} \leq 5 \times 10^{2} \mathrm{~mm}^{2} / \mathrm{s}$ ) for comparison. At the shutter opening period $T s \leq 103 \mathrm{~ms}$, $D_{32}$ remains constant value for each $v_{T}$ in the both methods. At $T s>103 \mathrm{~ms}, D_{32}$ decreases as increasing $v_{T}$ for $v_{T}<10^{3} \mathrm{~mm}^{2} / \mathrm{s}$ in the sedimentation method. As mentioned before, droplets coalesce easily when the silicone-oil viscosity $v_{T}$ is smaller, since the droplets float and move easily during sedimentation. On the other hand, $D_{32}$ increases with $v_{T}$ for $v_{T} \geq 10^{3} \mathrm{~mm}^{2} / \mathrm{s}$ in the hanging method. The captured droplets on the silicone-oil surface stay longer when the silicone-oil viscosity $v_{T}$ is larger, which causes the droplet coalescence on the oil surface.

Kurabayashi recommended that the desirable immersion liquid viscosity employed in the hanging method is $10^{3} \mathrm{~mm}^{2} / \mathrm{s}$ in order to suppress the captured droplet movements and aggregations. On the other hand, Rupe [13] suggested that the desirable immersion liquid viscosity employed in the sedimentation method is $1-10 \mathrm{~mm}^{2} / \mathrm{s}$, so that droplets immerse quickly.

As mentioned before, little effect of $v_{T}$ on droplet coalescence was observed when in the range of small $T s$ in both methods: the sedimentation method and the 


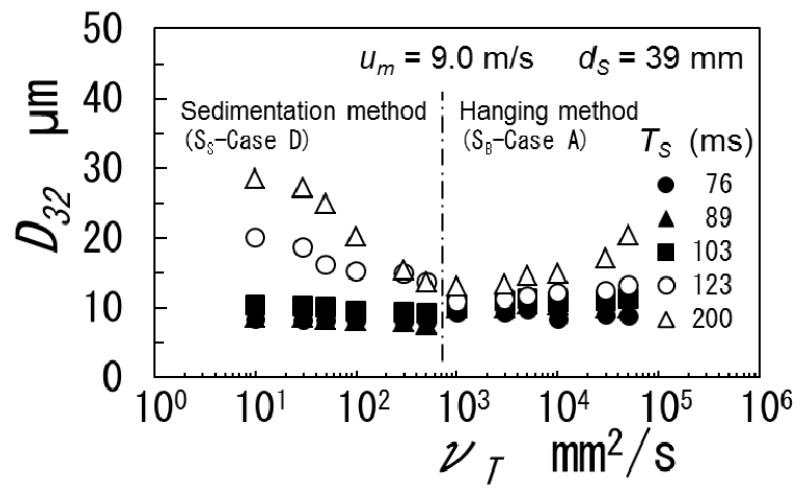

Figure 11: Influence of $D_{32}$ on silicone-oil viscosity $\nu_{T}$.

hanging method. The increase of $v_{T}$ is one of the factors for suppressing the effect of droplet coalescence in the range of large $T s$ in the sedimentation method.

\section{Conclusions}

In this study, the effects of the droplet coalescence and solution on the measurement accuracy focusing on the immersion liquid method using siliconeoil and water droplets generated by ultrasonic vibration were investigated. The obtained main results in this experiment as follows:

1. Captured droplets dissolve over time. The reduction in droplet number due to solution is slightly increased compared with the evaporation in the hanging method.

2. Using silicone-oil saturated with water as an immersion liquid, droplets stay longer than that using natural silicone-oil.

3. When the sampling tank is covered with a glass plate to avoid contact with ambient air (Case D), the effect of droplet solution is significantly reduced.

4. The sedimentation method cause more droplet coalescence than the hanging method.

5. Using the water saturated oil Type $\mathrm{S}_{\mathrm{S}}$ as the immersion liquid and the Case $\mathrm{D}$ as the measurement procedure, little effect of droplet coalescence and solution is observed in the both methods at $T \mathrm{~s} \leq 103 \mathrm{~ms}$.

\section{References}

[1] Hiroyasu, H., Kadota, T. and Arai, M., Supplementary Comments: Fuel Spray Characterization in Diesel Engines, Proc. of the Symposium on Combustion Modeling in Reciprocating Engines, pp. 369-408, 1980. 
[2] Ueda, T., Entrainment Rate and Size of Entrained Droplets in Annular Two-Phase Flow, Bulletin of the JSME, Vol. 22, No. 171, pp. 1258-1265, 1979.

[3] Al-Sarkhi, A. and Hanratty, T.J., Effect of Pipe Diameter on the Drop Size in a Horizontal Annular Gas-liquid Flow, Int. J. of Multiphase Flow, Vol. 28, pp. 1617-1629, 2002.

[4] Maeda, M., Hishida, K., Nakamura, K. and Ikai, S., Measurement of Particle Size, Velocity and Number Density in Polyphase Flow with an LDV Technique: On Signal Processing for Particle Sizing Using a Fringe Mode Type LDV Optical System, Trans. JSME 48(425), pp. 69-77, 1982.

[5] For example, Hurlburt, E. T. and Hanratty, T. J., Measurement of Drop Size in Horizontal Annular Flow with the Immersion Method, Experimental in Fluids, 32, pp. 692-699, 2002.

[6] Hiroyasu, H., Measurement of Atomization, J. of the Int. Comb. Engine Japan, Vol. 11, No. 127, pp. 105-115, 1972.

[7] Kurabayashi, T., Measurement of Spray Droplet Sizes and its Problems, J. of the Fuel Society of Japan, Vol. 53 No. 8, pp. 681-691, 1974.

[8] For example, Nukiyama, S., and Tanazawa, Y., An Experiment on the Atomization of Liquid by Means of an Air Stream (1st Report), Trans. JSME 4(14), pp. 128-135, 1938.

[9] Cousins, L. B. and Hewitt, G. F., Liquid Phase Mass Transfer in Annular Two Phase Flow: Droplet Deposition and Liquid Entrainment, UKAEA Report, AERE-R5657, 1968.

[10] Pogson, J. T., Roberts, J. H. and Waibler, P. J., An Investigation of the Liquid Distribution in Annular Mist Flow, Trans. ASME J. Heat Transfer, Vol. 92, No. 4, pp. 651-685, 1970.

[11] Whalley, P. B., Azzopardi, B. J., Pshyk, L. and Hewitt, G. H., Axial View Photograph of Waves in Annular Two Phase Flow, UKAEA Report, AERE-R8787, 1977.

[12] Tate, R. W., Immersion Sampling of Spray Droplets, AIChE J. 7(4), pp. 574-577, 1961.

[13] Rupe, J.K., A technique for the investigation of spray characteristics of constant flow nozzles, Third Symposium on Combustion, Flame and Explosion Phenomena, pp. 680-694, 1949. 